\title{
Poster: Do Open Resources Encourage Entry into the Millimeter Wave Cellular Service Market?
}

\author{
Fraida Fund, Shahram Shahsavari, Shivendra S. Panwar, Elza Erkip, Sundeep Rangan \\ Department of Electrical and Computer Engineering, NYU Tandon School of Engineering \\ \{ffund, shahram.shahsavari, panwar, elza, srangan\}@nyu.edu
}

\begin{abstract}
The resource usage model for millimeter wave bands has been the subject of considerable debate. The massive bandwidth, highly directional antennas, high penetration loss and susceptibility to shadowing in these bands suggest certain advantages to spectrum and infrastructure sharing. In particular, resources that are "open", such as unlicensed spectrum or a deployment of base stations open to all service providers, may offer greater gains in mmWave bands than at conventional cellular frequencies. However, even when sharing is technically beneficial (as recent research in this area suggests that it is), it may not be profitable. In this paper, both the technical and economic implications of resource sharing in millimeter wave networks are studied. Millimeter wave service is considered in the economic framework of a network good, and detailed network simulations are used to understand data rates, profit, and demand for millimeter wave service, with and without open resources. The results suggest that "open" deployments of neutral small cells that serve subscribers of any service provider encourage market entry by making it easier for networks to reach critical mass, more than "open" (unlicensed) spectrum would.
\end{abstract}

\section{CCS Concepts}

-Networks $\rightarrow$ Network economics; Mobile networks;

\section{INTRODUCTION}

The millimeter wave (mmWave) bands are the largest unlicensed bandwidths ever allocated, and regulatory agencies are moving quickly to open them for use. However, the specific regulatory scheme for these bands, particularly with respect to resource sharing, is yet undecided. Technical properties of mmWave bands favor spectrum and infrastructure (base station) sharing. Even when sharing improves consumers' quality of service, though, it may reduce the service provider's profits if it shifts demand to a competitor, or if it changes the market dynamics in a way that forces down the price. Early work in this area $[1,5,6]$ argues for sharing, but ignores the market dynamics of demand and competition.

Permission to make digital or hard copies of part or all of this work for personal or classroom use is granted without fee provided that copies are not made or distributed for profit or commercial advantage and that copies bear this notice and the full citation on the first page. Copyrights for third-party components of this work must be honored. For all other uses, contact the owner/author(s).

MobiCom'16 October 03-07, 2016, New York City, NY, USA

(C) 2016 Copyright held by the owner/author(s).

ACM ISBN 978-1-4503-4226-1/16/10.

DOI: http://dx.doi.org/10.1145/2973750.2985272
In this work we model the strategic decisions of wireless service providers building out mmWave networks with or without open resources. We apply economic models of network goods [2] to mmWave cellular networks, and use simulations to quantify the effect of increasing network size, i.e., how a subscriber's data rate changes as the mmWave service provider increases its spectrum holdings, base station deployments, and market share. Using the concept of critical mass [3], we investigate the demand for a mmWave network service under different "open resource" scenarios.

\section{NETWORK EXTERNALITIES}

Consider mmWave network service as a network good [2], with varying demand and resources. Subscribers benefit from an indirect positive network externality: a large network service provider (NSP) with more subscribers (higher density of UEs) will build a denser deployment of base stations (BSs) and/or purchase more spectrum. Using the simulations described in [4], we find the effect on fifth percentile data rates of increasing network size $n$, for three scenarios: (a) No open resources: An NSP scales its spectrum licenses and BSs according to the number of subscribers. Network size, $n$, is the normalized demand for service (scaled to the range $[0,1])$, but is also a scaling factor on the BS density and bandwidth of the NSP. (b) Open BS deployment: There is a preexisting deployment of open association small cells, operated by a coalition of service providers or by a third party. Network size, $n$, refers to demand for service and is also a scaling factor on the bandwidth of the NSP, but the BS density of the NSP is constant and equal to the size of the "open" deployment for all $n$. (c) Open spectrum: Spectrum is unlicensed and may be used by any NSP. Here, $n$ refers to demand for service and is also a scaling factor on the BS density of the NSP. The bandwidth of the NSP is constant, equal to the full unlicensed bandwidth for all $n$.

Fig. 1(a) shows simulation results for a mmWave and a conventional microwave cellular scenario. The overall effect on fifth percentile data rates of increasing $n$ is piecewise linear, with the slope depending on the open resources and on whether the NSP has captured a small portion of the market (small $n$ ) or more of it (large $n$ ). We also note differences between mmWave and microwave networks. First, uncoordinated spectrum sharing improves mmWave user rate, but has a negative effect on microwave networks. Second, in microwave networks, the fifth percentile rate starts increasing for very small $n$. In mmWave networks, when there is no BS sharing, the fifth percentile rate remains flat for small $n$. This is due to the increased path loss at mmWave frequencies, requiring a denser deployment of $\mathrm{BSs}$ for coverage. 


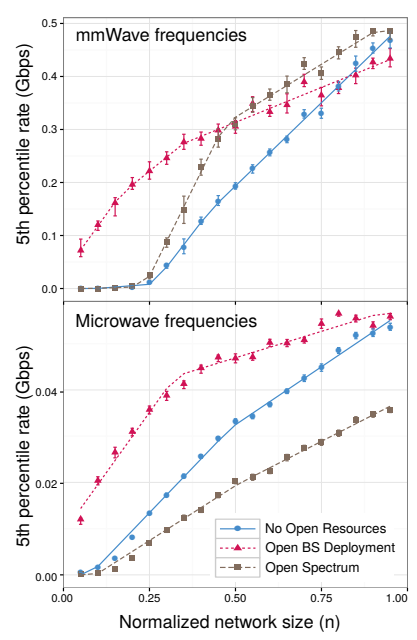

(a) We derive the network externalities function from the effect of increasing network size on fifth percentile rates.
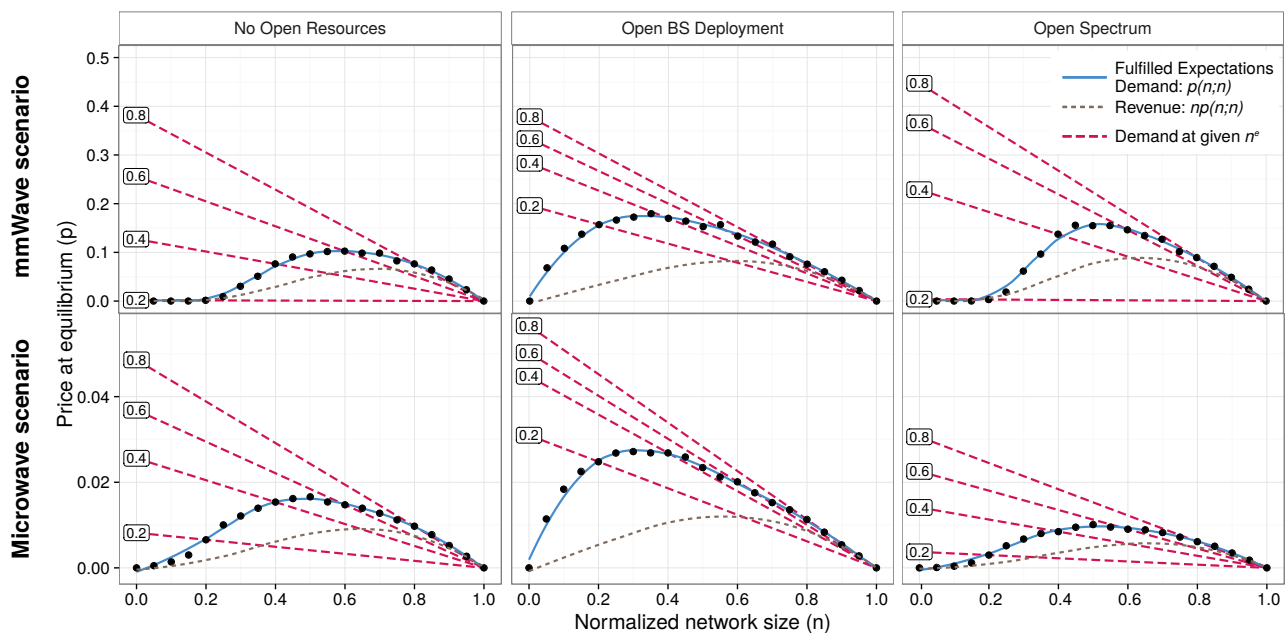

(b) The demand curves for mmWave service for a range of expected network sizes $n^{e}$, fulfilled expectations demand curve where $n=n^{e}$, and NSP revenue. In the mmWave scenario, slow initial growth makes it difficult to reach critical mass in the case of no open resources and open spectrum, while with an open BS deployment there is robust demand and growth even at small network sizes.

\section{EVOLUTION OF DEMAND}

Given these network externalities, we consider the effect of "open" resources on new service offerings, where the provider is concerned with establishing a stable market presence. Using a standard utility model for economics of network goods [2], we consider consumers whose utility for network service is $u(\omega, n, p)=\omega h(n)-p$, where $p$ is the price of service, $n$ is the network size, and $h(n)$ is the network externalities function derived from the slopes of the lines in Fig. 1(a). The taste parameter $\omega$ is distributed uniformly on the interval $[0,1]$ among the set of consumers. Fig. 1(b) shows the fulfilled expectations demand curve for the network (constructed as in [4]), which gives the size of the network that could be supported at equilibrium for a given price.

The slope of this curve at small network sizes determines how easily the network will reach a stable nonzero equilibrium [3]. Under perfect competition, with marginal cost $c$, equilibria occur at $n=0$ and at the points $n^{\prime}$ and $n^{\prime \prime}$ where the fulfilled expectations demand curve intersects the horizontal line $p=c$, and $n=0$ and $n=n^{\prime \prime}$ are stable equilibria. When the fulfilled expectations demand grows quickly from $n=0$, it is easier to reach the stable equilibrium at $n^{\prime \prime}$.

From Fig. 1(b), we find that in mmWave networks without an open deployment of BSs, the demand does not begin to grow until the network is moderately large, making it difficult for a new provider to reach critical mass. This is due to the propagation characteristics of mmWave bands, which require a denser deployment of BSs to provide coverage. With an existing deployment of "open" small cells, there is robust demand even at small network sizes, which encourages growth. Open spectrum increases demand and revenue once the network is sufficiently large, but does not have a similarly encouraging effect on market entry. In conventional microwave networks, we see the opposite: demand begins to grow even at small values of $n$, and unlicensed spectrum has a negative effect on demand and revenue.

\section{CONCLUSIONS}

We have connected economic models for cellular network service providers and subscribers, to detailed simulations of
mmWave networks. Our results suggest that based purely on a small NSP's ability to reach a stable equilbrium, an open BS deployment could ease the barrier to entry for cellular network providers who are considering adding mmWave service, encouraging new service offerings. However, this relies on a third party having invested in BSs, potentially ahead of demand if there are no mmWave NSPs in the market yet and the BSs are only useful for mmWave service. Open spectrum increases demand and revenue for moderate/large-sized mmWave networks, and relies only on regulators releasing spectrum for unlicensed use in cellular systems, but does not have a similarly encouraging effect on market entry.

\section{ACKNOWLEDGMENTS}

This work was supported by the National Science Foundation (Grants No. 1547332, 1302336, and the GRFP), the New York State Center for Advanced Technology in Telecommunications (CATT), and NYU WIRELESS.

\section{REFERENCES}

[1] F. Boccardi, H. Shokri-Ghadikolaei, G. Fodor, E. Erkip, C. Fischione, M. Kountouris, P. Popovski, et al. Spectrum pooling in mmwave networks: Opportunities, challenges, and enablers. arXiv:1603.01080, 2016.

[2] N. Economides. The economics of networks. International journal of industrial organization, 14(6):673-699, 1996.

[3] N. Economides and C. P. Himmelberg. Critical mass and network size with application to the US fax market. NYU Stern School of Business EC-95-11, 1995.

[4] F. Fund, S. Shahsavari, S. S. Panwar, E. Erkip, and S. Rangan. Spectrum and infrastructure sharing in millimeter wave cellular networks: An economic perspective. arXiv:1605.04602, 2016.

[5] A. K. Gupta, J. G. Andrews, and R. W. Heath Jr. On the feasibility of sharing spectrum licenses in mmwave cellular systems. arXiv:1512.01290, 2015.

[6] M. Rebato, M. Mezzavilla, S. Rangan, and M. Zorzi. Resource sharing in $5 \mathrm{G}$ mmwave cellular networks. arXiv:1603.02651, 2016. 